“(C) 2015 IEEE. Personal use of this material is permitted. Permission from IEEE must be obtained for all other uses, in any current or future media, including reprinting/republishing this material for advertising or promotional purposes, creating new collective works, for resale or redistribution to servers or lists, or reuse of any copyrighted component of this work in other works." 


\title{
Path Planning with Stability Uncertainty for Articulated Mobile Vehicles in Challenging Environments
}

\author{
Mohammad Norouzi, Jaime Valls Miro, Gamini Dissanayake and Teresa Vidal-Calleja
}

\begin{abstract}
This article proposes a probabilistic approach to account for robot stability uncertainty when planing motions over uneven terrains. A novel probabilistic stability criterion derived from the cumulative distribution of a tip-over metric is introduced that allows a safety constraint to be dynamically updated by available sensor data as it becomes available. The proposed safety constraint authorizes the planner to generates more conservative motion plans for areas with higher levels of uncertainty, while avoids unnecessary caution in well-known areas.The proposed systematic approach is particularly applicable to reconfigurable robots that can assume safer postures when required, although is equally valid for fixed-configuration platforms to choose safer paths to follow. The advantages of planning with the proposed probabilistic stability metric are demonstrated with data collected from an indoor rescue arena, as well as an outdoor rover testing facility.
\end{abstract}

\section{Motivation And Related Work}

One of the most difficult problems of navigation over unstructured and unforgiving environments is how to address the uncertainties emanating from imperfect actuators and poor environmental sensor information. Several approaches have been developed in the literature to deal with uncertainties in the input data and system model parameters. For instance, path following with uncertainty has been studied by the control community. A Kalman-based active observer controller for the path following of wheeled mobile robots subject to non-holonomic constraints is presented in [1]. The effect of external disturbances, general model errors, and uncertainties present in the system are reduced by adding an extra state (the "active state") to the controller design. The effectiveness of the proposed path-following controller was evaluated via simulation results for a wheelchair robot following a straight line and a circular path. Path following controllers based on a Lyapunov feedback linearisation have also been proposed to make the controller robust to modelling uncertainty, e.g. for articulated manipulators where experimental results of a $4 \mathrm{DoF}$ manipulator with revolute and linear actuated links were presented moving the endeffector along a circular path [2].

Other authors have looked at the problem of incorporating uncertainty at the planning stage, e.g. by considering variations in the $2.5 \mathrm{D}$ terrain elevation data and localisation errors, as described in [3] for an articulated wheeled mobile robot. A conservative path planning approach is adopted that considers terrain measurement uncertainty, where a set

\footnotetext{
All authors are with the Faculty of Engineering
and IT, University of Technology Sydney (UTS), Sydney NSW 2007, Australia. \{mohammad.norouzi, jaime.vallsmiro, gamini.dissanayake, teresa.vidalcalleja\}@uts.edu.au
}

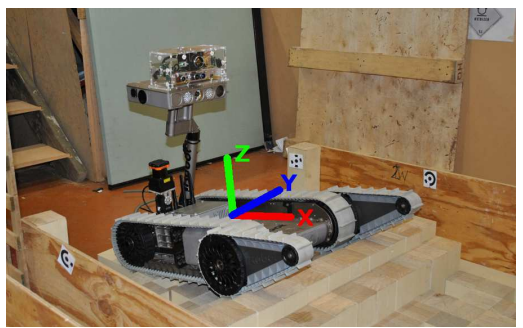

Fig. 1: The iRobot PackBot Explorer robot with a 1 DoF arm, pan-tilt sensor unit and two small front sub-tracks (flippers).

of potential worst-case robot configurations at boundary locations in the terrain are examined to make sure that the vehicle would remain stable for a given variance in the elevation map. If any posture in this set is proven unstable, the corresponding location in the map will be regarded as untraversable. To address the localisation uncertainty for a given path, all points along the path within a distance proportional to the assumed robot localisation uncertainty are examined given all possible configurations. A point in the terrain would be considered as a feasible point for path finding purposes only if all configurations in the overall search have been proven to be stable. The output of this brute-force approach is a simple failure or success, with no concern for the probability of a tip-over instability.

This article looks at the challenging problem of global path planning over ruggedised terrains by formally accounting for stability uncertainty in the process. A novel probabilistic stability metric based on the uncertainty analysis technique described in [4] is introduced. The proposed criterion is employed to progress the deterministic stable path planning strategy described in [5], proven to be particularly suitable for search and rescue missions, with the goal of improving robot navigation safety in scenarios where the model of the system and the sensory data available to the robot may be imperfect. In [5], the force angle (FA) stability measure [6] was employed to evaluate the stability of the rover along the path. The FA margin is deterministically defined by the position of the robot's centre of mass $(\mathrm{CM})$ and the contactpoint $(\mathrm{CP})$ interaction with the terrain, which form a convex area called "support polygon" (SP). The main drawback of employing deterministic constant stability margins to path planning is that while producing safer paths with larger, more conservative stability margins, they may also easily end up being overly restrictive, filtering out many probable pathways, while on the other hand planning on the boundary of tip-over could easily jeopardise stability if uncertainties 


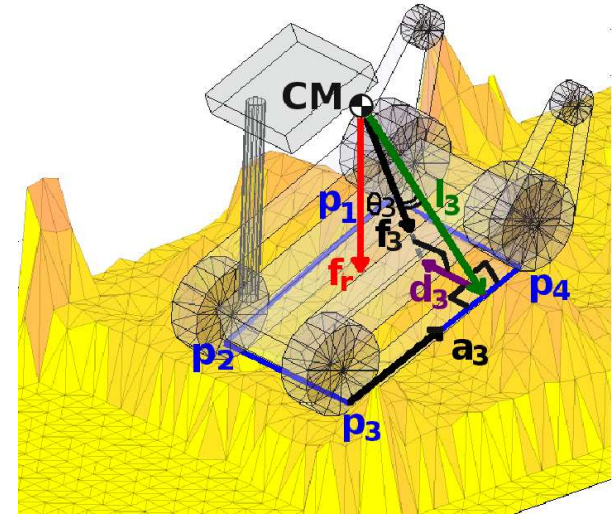

Fig. 2: The 3D FA stability measure for $n=4$ and $i=3$. (CM's position has been shifted up and vectors scaled for easier visualization). The FA measure can be intuitively described as the effect of the forces over CM projected on the supporting convex area defined by the contact-points between the vehicle and terrain e.g. $\beta_{3}=\theta_{3}\left\|\mathbf{d}_{\mathbf{3}}\right\|\left\|\mathbf{f}_{\mathbf{3}}\right\|$.

are present.

The proposed probabilistic approach allows to search paths with a minimum "safety confidence" instead, so that model uncertainties can be taken into consideration when finding paths, instead of resorting to restrictive fixed minimum safe margins. The advantages of planning with probabilistic stability will be demonstrated with a model of the Packbot robot shown in Fig. 1 through comprehensive simulations in a mock-up Urban Search and Rescue (USAR) arena an data from a quasi-outdoor rover testing facility at the University of Toronto Institute for Aerospace Studies (UTIAS) [7].

\section{OVERVIEW OF STABILITY ANALYSIS}

To anticipate the stability measure, works like [8] have considered an ideal support polygon (ISP) for the vehicle, i.e. contact points are assumed to be fixed under the sprockets of the robot. It has been illustrated in the literature [5] how this is a strong assumption for the case of highly unstructured terrains, where contact points can lay anywhere along the robot's track and in general describe a variable support polygon (VSP). Hence, no ISP is assumed in the following sections.

\section{A. Force Angle Stability Metric}

The example in Fig. 2 describes the FA derivations for the third tip-over axis with a vehicle exhibiting four CPs in a given terrain configuration. The line joining two consecutive CPs $\left(\mathbf{p}_{\mathbf{i}}\right.$ and $\mathbf{p}_{\mathbf{i}+\mathbf{1}}$ ) forms the tip-over axes $\mathbf{a}_{\mathbf{i}}$. The shortest vector between the CM and each $\mathbf{a}_{\mathbf{i}}$ is referred to as $\mathbf{l}_{\mathbf{i}}$. The FA stability margin [6] takes into account $\mathbf{d}_{\mathbf{i}}$, the distance between the projected CM to the ith tip-over axis $\mathbf{a}_{\mathbf{i}}$, and $\mathbf{f}_{\mathbf{i}}$ the component of the effective net force $\mathbf{f}_{\mathbf{r}}$ which acts about the axis $\mathbf{a}_{\mathbf{i}}$. It also considers the angle $\theta_{i}$ between $\mathbf{f}_{\mathbf{i}}$ and the tip-over axis normal $\mathbf{l}_{\mathbf{i}}$. Assuming the SP is composed of $n$ CPs, the FA stability measure about each $\mathbf{a}_{\mathbf{i}}$ can be formulated as

$$
\beta_{i}=\theta_{i}\left\|\mathbf{d}_{\mathbf{i}}\right\|\left\|\mathbf{f}_{\mathbf{i}}\right\|, \quad i=\{1, \ldots, n\}
$$

The final FA margin would be the minimum $\beta_{i}$ i.e. $\beta=$ $\min \left(\beta_{i}\right), \quad i=\{1, \ldots, n\}$.

\section{B. Robot Model}

Fig. 1 shows the multi-tracked iRobot Packbot robot model and its coordinate frame convention that was employed in this paper to validate the simulation results. The mechanical structure consists of a skid-steer vehicle base, flippers (two synchronised small sub-tracks in the front) and an arm that carries a 2D pan-and-tilt unit equipped with several cameras and lights. It is clear that for these types of robots the arm and/or flippers angles $\left(\phi_{a}\right.$ and $\left.\phi_{f}\right)$ will significantly affect the location of the CM. Moreover, when the flippers are in contact with the terrain they change the shape of the SP, which in turn has a more significant effect on the stability of the robot. More details about the robot's kinematic model and the effect of the mass distribution can be found in [5].

\section{Contact-Points Prediction}

The robot-terrain prediction algorithm is based on the mathematical description of the robot in the Open Dynamics Engine (ODE) [9], a widely used physical rigid body dynamics simulator. A 3D model of the terrain has been constructed from the ranging data measured with the RGB-D camera situated on the head of the robot. The CP derivation scheme is predicated on calculating the projection of the robot's geometric underside on the points defining the terrain underneath. Under the assumption of quasi-static equilibrium, the simulator predicts the behaviour of the robot under the influence of gravitational forces for a given pose and posture configuration to extract the SP. This process is described in detail in [5].

\section{UnCertainty Analysis and Planning Method}

The probabilistic stability metric calculation, the definition of the proposed safety confidence and it's use in the context of path planning which form the novel contribution of this paper are described in the following sections.

\section{A. Transformation of Means and Covariance}

The probabilistic approach for uncertain stability analysis is detailed in [4]. For completeness, this section will quickly summarise the aspects most relevant to the novel proposition in this work. The general problem can be expressed as follows: for an $\mathrm{n}$-dimensional input vector $\mathbf{x}$ with given mean $\hat{\mathbf{x}}$ and covariance $\mathbf{P}_{x x}$, what would be the mean $\hat{\mathbf{y}}$ and covariance $\mathbf{P}_{y y}$ of an m-dimensional random variable vector $\mathbf{y}$, where $\mathbf{y}$ is related to $\mathbf{x}$ by a non-linear transformation $\mathbf{y}=$ $g[\mathbf{x}]$. For the system hereby considered, the arm and flipper angles $\left(\phi_{a}, \phi_{f}\right)$ that determine the posture of the robot, the 3D model of a given terrain and the robot's position on it constitute the input parameters, i.e. $\mathbf{x}_{37 \times 1}=\left(\phi_{a}, \phi_{f}, r x, r y, y a w, 32 \times\right.$ terrain sections). The output vector includes a list with (up to) four $\mathrm{CPs}$, the $\mathrm{CM}$ and the FA stability measure, i.e. $\mathbf{y}_{16 \times 1}=\left(4 \times\left(C P \_x, C P_{-} y, C P \_z\right),\left(C M_{-} x, C M_{-} y, C M_{\_} z\right), \beta\right)$. 


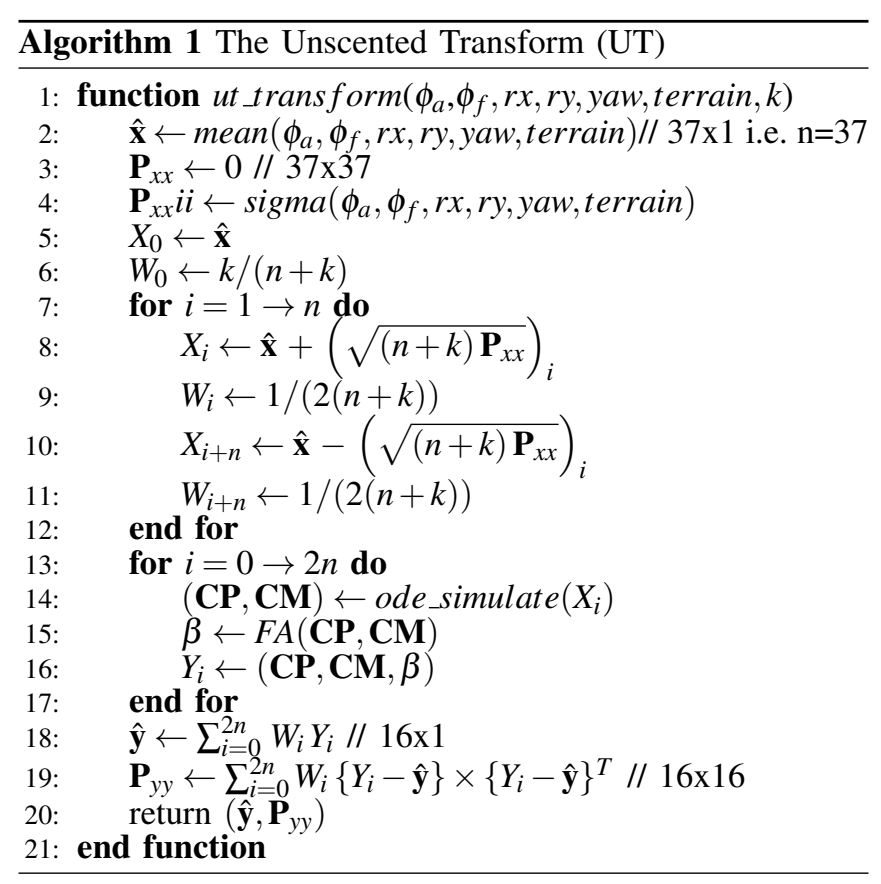

Given the highly non-linear nature of $g[$.$] , Taylor series$ approximation and general error propagation [10] are not applicable to enumerate $\hat{\mathbf{y}}$ and $\mathbf{P}_{y y}$. Standard Monte Carlo (SMC) [11] is a proven iterative algorithm to estimate probability density functions of a general system's output response from a large set of random inputs. Hence, by introducing perturbations to the input parameters, ODE simulations can be carried out and $\beta$ subsequently calculated. The tendency to bigger input sets to attain more accurate distributions makes SMC computationally expensive. The structured unscented transform (UT) [12] has been proposed in the literature to address this issue, and was employed in this work to speed up the transformation of means and covariances. The overall technique as applied to this work, summarised in Alg. 1, intelligently simulates the SMC method by choosing a deterministic set of inputs instead of a vast random sample population.

It is important to note that while only the FA distributions $\left(\beta_{\mu}, \beta_{\sigma}\right)$ are exploited for path planning purposes in this work, the output vector $\mathbf{y}$ also provides probabilistic information about the robot's CPs and CM. It is envisaged that it may well be possible to take advantage of these useful statistics in other stability metrics, or for other purposes (e.g. computer graphics rendering applications).

\section{B. Probabilistic Stability Metric}

Assuming a standard normal distribution $N(0,1)$ for $\beta$, the cumulative distribution function $(\mathrm{CDF})$ is formulated as:

$$
\Phi(x)=\frac{1}{\sqrt{2 \pi}} \int_{-\infty}^{x} \mathrm{e}^{-\frac{t^{2}}{2}} \mathrm{~d} t
$$

This function describes the probability that $\beta$ will be found at a value less than or equal to $x$, where $\Phi(-\infty)=$ $0 \%, \Phi(0)=50 \%$ and $\Phi(\infty)=100 \%$. For a generic normal

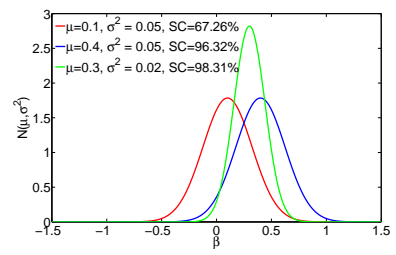

(a) $\beta_{\mu}>0$

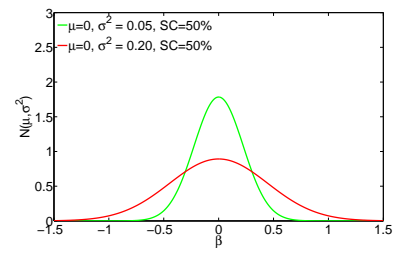

(b) $\beta_{\mu}=0$
Fig. 3: Possible PDFs for $\beta$ and the corresponding $S C$ values.

distribution $N\left(\beta_{\mu}, \beta_{\sigma}^{2}\right)$ for $\beta$, the cumulative distribution function can be transformed by

$$
F\left(x, \beta_{\mu}, \beta_{\sigma}\right)=\Phi\left(\frac{x-\beta_{\mu}}{\beta_{\sigma}}\right)
$$

Therefore $F\left(0, \beta_{\mu}, \beta_{\sigma}\right)$ will indicate the probability that $\beta$ will assume negative values (i.e. a tip-over is in progress). We can now define a "Safety Confidence" margin $(S C)$ to encapsulate our confidence in the stability prediction as

$$
S C(\beta)=\left(1-F\left(0, \beta_{\mu}, \beta_{\sigma}\right)\right) \times 100
$$

To intuitively understand the meaning of $S C$ the example in Fig. 3a is provided. The graph illustrates possible probability density functions (PDF) for $\beta$, and the corresponding values for $S C$, based on three different robot postures at a given location on a terrain. Although the mean value of the green distribution is smaller than the blue one, a larger $S C$ value indicates more certainty in this configuration. A conservative fixed large $\beta$ will unnecessarily push the robot away from many potentially feasible trajectories. On the other hand, critically small safety margins may put the robot in jeopardy, particularly when traversing highly challenging terrains (e.g. stairs or rubble). By employing the proposed $S C$ margin instead, the system can benefit form a dynamic safety boundary that represents reliability in the output predictions.

For the special case when the mean value is exactly zero, the SC calculation would be independent of $\sigma^{2}(S C=50 \%$ always, as illustrated by Fig. 3b). In this case, although both distributions result in the same value for $S C$, for stability purposes a distribution with smaller $\sigma^{2}$ should be preferred (green curve in this example), indicating that the true $\beta$ is generally expected to be closer to zero and away from negative tip-over instability. Therefore, for the special case when $\mu=0, S C$ will be multiplied by $\left(1-\sigma^{2}\right)$ to lean towards configurations with smaller covariances.

\section{High Visibility Probabilistically Stable Path Planning}

The fundamental motivation behind the proposal in this paper is the ability to identify safe paths that can also accommodate uncertainty in the stability of the proposed manoeuvres in a probabilistic manner. To that end, changes are proposed to the deterministic technique described in [5] where the benefits of an optimum stable high-visibility path planner for rescue operations were heralded. Given space constraints only an intuitive description of the methodology 


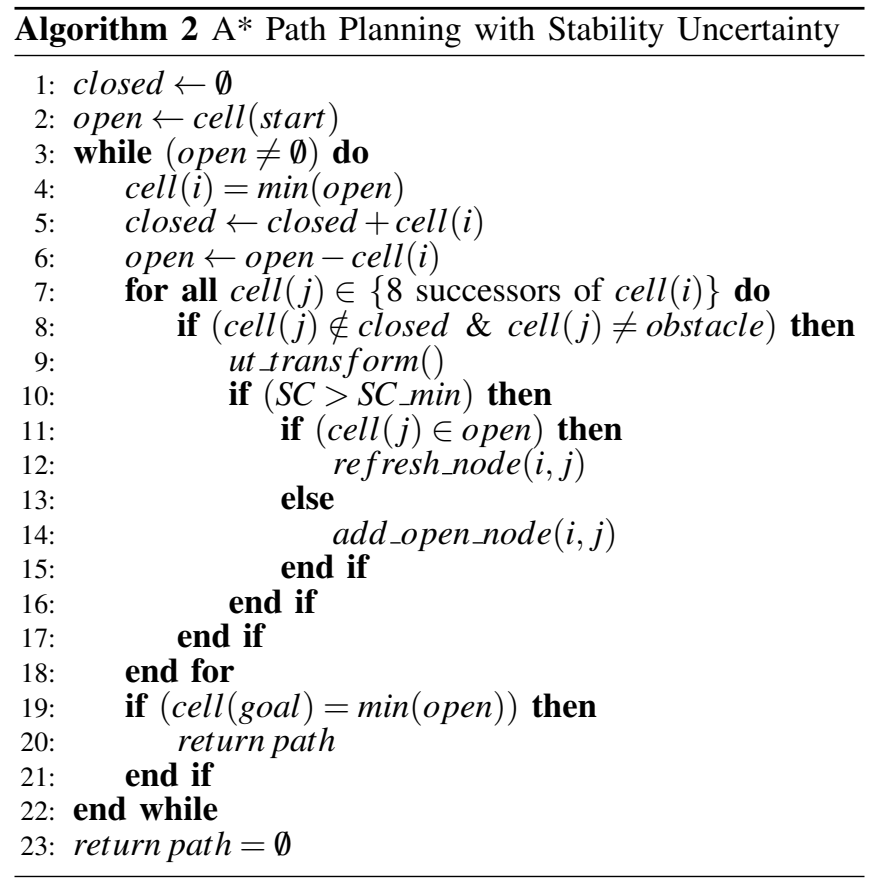

in the context of that work is provided so that the novel proposition and the comparison results in the following sections can be fully appreciated.

The key contribution on the algorithm in [5] was the introduction of a stability constraint to a cost-based $\mathrm{A}^{*}$ planner. Essentially, the stable $\mathrm{A}^{*}$ algorithm first examines the stability of the robot when opening a new search node at a new location with a given configuration. The node is considered stable if $\beta$ being larger than some nominal $\beta_{\text {min }}$ is satisfied. The present proposal, abstracted by Alg. 2, takes into account $S C$ as described by (4) through the ut_transform () function (Alg. 1), effectively transforming the fixed stability constraint $\left(\beta>\beta_{\text {min }}\right)$ into a minimum confidence threshold $\left(S C>S C_{\text {min }}\right)$ representative of the certainty in the stability prediction.

\section{Simulation Results}

The effectiveness of the approach has been evaluated using two terrain data sets, and compared to the deterministic stable path planning described in [5]. The USAR test arena is chosen to investigate the performance of the technique in an indoor setting with distinctive features such as stairs, rubble etc., whereas the UTIAS arena is an example of a larger outdoor scenario. In both instances, the robot is expected to come up with configurations aimed at keeping the arm as high as possible to achieve the best possible field of view while satisfying the constraints imposed by the corresponding algorithms $\left(\beta_{\min }\right.$ or $\left.S C_{\min }\right)$.

\section{A. Planning on a USAR Arena}

The UTS mock-up rescue arena consists of a $6 m \times 8 m$ reconfigurable rectangle space with a ramp, a flight of stairs, open space and re-arrangeable blocks of step-fields. A small section is captured by Fig. 1. The 3D model of the terrain

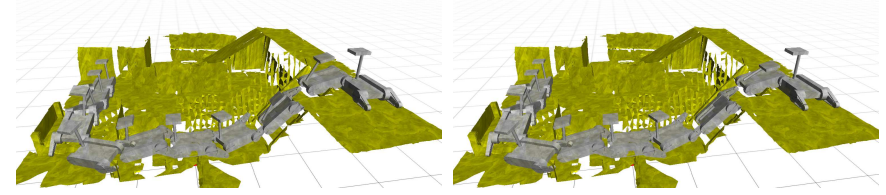

(a) Path with $\beta_{\min }=0.05$.

(b) Path with $S C \_$min $=50 \%$.

Fig. 4: Planning based on the minimum safety margin and stability confidence in the USAR arena. Planning based on $S C$ generates safer postures over stairs $\left(\phi_{a}=0^{\circ}\right.$ in b) when compared to the deterministic approach $\left(\phi_{a}=20^{\circ}\right.$ in a).

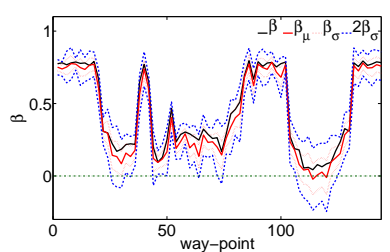

(a) $\beta\left(\beta_{\min }=0.05\right)$.

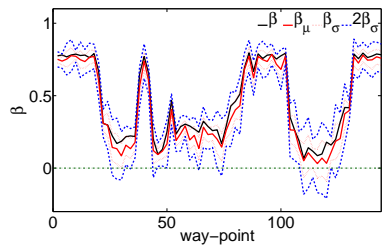

(c) $\beta\left(S C_{\min }=50 \%\right)$.

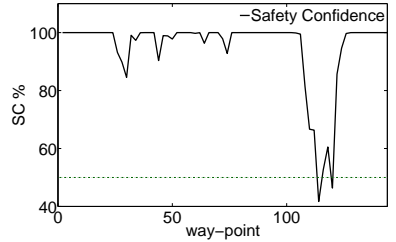

(b) $S C\left(\beta_{\min }=0.05\right)$

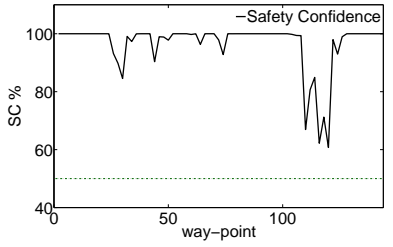

(d) $S C\left(S C_{\min }=50 \%\right)$
Fig. 5: Comparison of $S C$ and $\beta$ over the trajectories depicted in Fig. 4 with $\beta_{\min }=0.05$ and $S C_{\min }=50 \%$. The horizontal dark green dash-dot lines indicate reference points where $\beta=0$ or $S C_{\min }=50 \%$ (likewise in Fig. 8).

was built off-line by scan matching of the RGB-D data logs when manually operating the robot over the terrain at low speeds. In order to make a fair comparison between the two planners, a pre-processing step was first applied to the terrain model to label out obvious untraversable areas, e.g. walls and markedly steep slopes. Two sets of experiments of planning based on varying allowable boundaries for $\beta_{\text {min }}$ and $S C_{\text {min }}$ are studied in order to highlight the advantages of the probabilistic approach in generating safer and more optimal posture planning.

In the first scenario, planners are set to find a path from the top left corner of the USAR arena with a minimum possible $\beta_{\text {min }}=0.05$ and $S C_{\text {min }}=50 \%$ to the goal at the bottom right corner. The value of $\beta_{\text {min }}=0.05$ was obtained experimentally as the border of stability when the robot was sitting on the $35^{\circ}$ ramp of the arena, with the nominal configuration $\left(\phi_{a}=90^{\circ}, \phi_{f}=90^{\circ}\right)$. A positive $\beta_{\mu}$ is the only requirement to achieve $S C_{\min }=50 \%$, consequently the minimum allowable safety confidence is assumed to be $50 \%$.

The results are depicted in Fig. 4, where Fig. 4a and $4 \mathrm{~b}$ illustrate the outcomes of the shortest deterministic and probabilistically stable paths respectively. Only a limited 


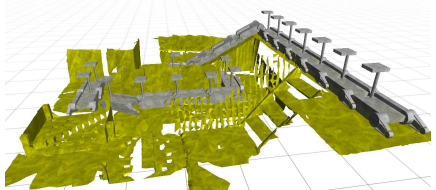

(a) Path with $\beta_{\min }=0.20$.

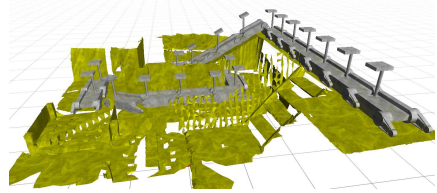

(b) Path with SC_min $=70 \%$.
Fig. 6: Planning based on a comfortable safety margin and stability confidence in the USAR arena. Planning based on $S C$ generates postures with better visibility over the ramp $\left(\phi_{a}=50^{\circ}\right.$ in b) when compare to the deterministic approach $\left(\phi_{a}=0^{\circ}\right.$ in a).

number of the robot poses are shown in the figure for clarity. In both instances the final paths traverse through the stepfields and the stairs, and the robot configurations over both trajectories end up being quite similar (except on the stairs, way-points around $100-130$ in Fig. 5, discussed below).

The comparison of $S C$ and $\beta$ over these trajectories are depicted in Fig. 5. The mean value of the stability measure obtained using the UT transform $\beta_{\mu}$ at each instant is depicted in red, with the standard deviation $\sigma(68 \%)$ and $2 \times \sigma(95 \%)$ around the mean depicted in dashed red and blue in Fig. 5a and 5c. The Fig. 5b and 5d illustrate the corresponding $S C$ measures of the resulting two paths.

It can be seen how by setting an arbitrary lower boundary $\left(\beta_{\min }=0.05\right)$ the deterministic planner's limited concern about the instantaneous value of $\beta$ results in paths with instances where, although as shown in Fig. 5a $\beta$ is computed to be always larger than $\beta_{\min }=0.05$, in some places the corresponding $\beta_{\mu}$ is actually negative $(S C<50 \%)$, indicating a high risk for tip-over instability as illustrated in Fig. 5b. This happens for instance over the stairs (way-points around 117), where $\beta_{\mu}$ is indeed less than 0.05 .

On the other hand, as depicted by Fig. 5d, a planner considering an $S C_{\min }=50 \%$ might end up with instances when $\beta_{\mu}$ is less than 0.05 in some places (see Fig. 5c). However, SC remaining over the threshold of $50 \%$ only requires a positive $\beta_{\mu}$, which is comfortably achieved by the planner generating postures with lower sensor head heights (e.g. $\phi_{a}=0^{\circ}$ over the stairs section depicted in Fig. 4b), compared to the resulting postures $\left(\phi_{a}=20^{\circ}\right)$ of a deterministic planner when $\beta_{\text {min }}=0.05$ (Fig. 4a). This example clearly shows how the probabilistic approach tends towards more conservative paths stability-wise than a deterministic planner in areas where uncertainty escalates.

In the following example the safety margin and stability confidence are increased to $\beta_{\min }=0.20$ and $S C_{\min }=70 \%$ respectively. Both criteria will now filter out the stairs and step-fields, tending towards a safer but longer path to the goal through the ramp, as shown in Fig. 6. Planning based on $\beta_{\min }=0.20$ has configured the robot with $\left(\phi_{a}=0^{\circ}\right)$ over the ramp. Yet given the higher certainty of the map over the ramp (as opposed to more rugged terrain sections), the probabilistic planner with $S C_{\min }=70 \%$ can satisfy the

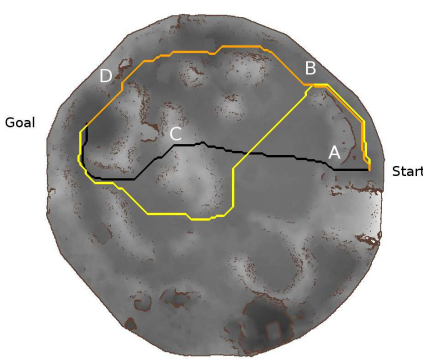

(a) Planning according to $\beta_{\text {min }}$.

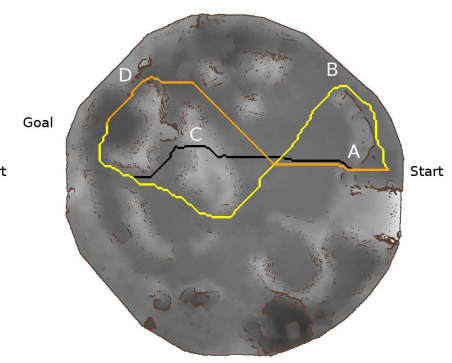

(b) Planning according to $S C_{\text {min }}$.
Fig. 7: Planning in the UTIAS arena. The paths with $\beta_{\min }=$ 0.05 and $S C_{\min }=50 \%$ are illustrated in black, paths with $\beta_{\min }=0.10$ and $S C_{\min }=70 \%$ are depicted in orange and yellow trajectories showing the paths with $\beta_{\min }=0.20$ and $S C_{\min }=90 \%$.

stability constraint with better field of view configuration $\left(\phi_{a}=50^{\circ}\right)$ for the same area.

\section{B. Planning on a Planetary Rover Testing Arena}

The proposed algorithm is tested with 3D terrain data collected from the quasi-outdoor UTIAS rover testing arena [7]. The UTIAS testing facility consists of a large dome structure, which covers a workspace area $40 \mathrm{~m}$ in diameter. These datasets are available online and for more information, the reader is referred to [7]. The data is used to study the outcomes of planning longer paths with different values for $\beta_{\min }$ and $S C_{\min }$. Results in Fig. 7 show how when the stability constraint is reasonable medium value, the statistical approach can find more effective and shorter path than deterministic technique (the path shown in orange). Moreover statistical analysis on the outcome of planning based on minimum allowable $\beta_{\min }=0.05$ has resulted in a $S C<50 \%$ in some places over the trajectory which indicates a high risk for a tip-over instability.

The outcomes of a planner based on different deterministic stability margins are shown in Fig. 7a where the path with lowest allowable safety margin $\beta_{\min }=0.05$ is illustrated in black, and paths with $\beta_{\min }=0.10$ and $\beta_{\min }=0.2$ are depicted in orange and yellow respectively. Gray-scale colour coding indicates height of the terrain from 0 to $2.76 \mathrm{~m}$. A preprocessing algorithm based on terrain gradients was first applied to the model to label out obviously untraversable steep slopes, shown in dark Brown. Given the space limitations, only the uncertainty analysis results of the first two trajectories are shown in Fig. 8, where the mean values of the stability measure using the UT transform at each instant are depicted in red, the standard deviation $\sigma(68 \%)$ and $2 \times \sigma$ $(95 \%)$ around the mean are depicted in dashed red and blue in Fig. 8a and 8c. Fig. 8b and 8d illustrate the corresponding $S C$ measures. In the same way Fig. $7 \mathrm{~b}$ shows the effect of different values of $S C_{\min }$ on the planner, where black, orange and yellow illustrate trajectories with $S C \_\min =50 \%$, $S C \_$min $=70 \%$ and SC_min $=90 \%$ respectively. The corresponding uncertainties are shown in Fig. 8. It can be seen 
(a) $\beta\left(\beta_{\min }=0.05\right)$.

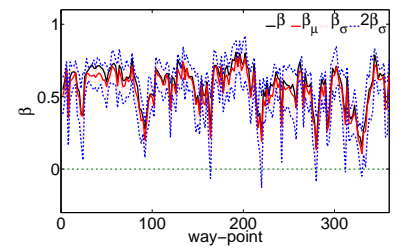

(c) $\beta\left(\beta_{\min }=0.10\right)$.

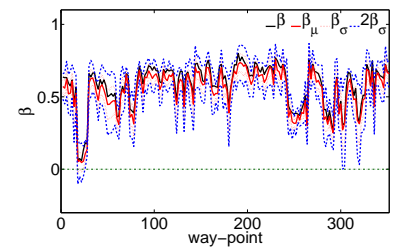

(e) $\beta\left(S C_{\min }=70 \%\right.$.)

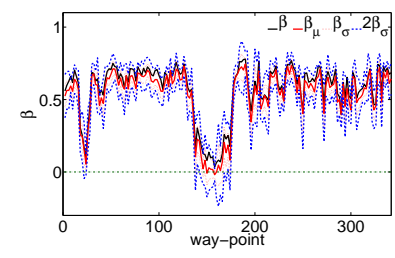

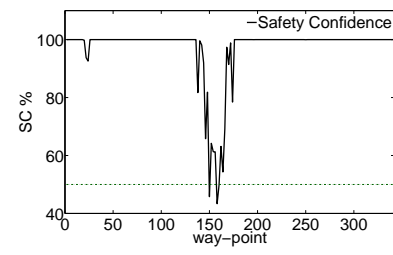

(b) $S C\left(\beta_{\min }=0.05\right)$.

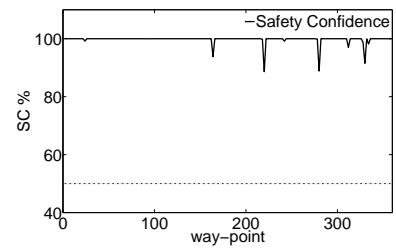

(d) $S C\left(\beta_{\min }=0.10\right)$.

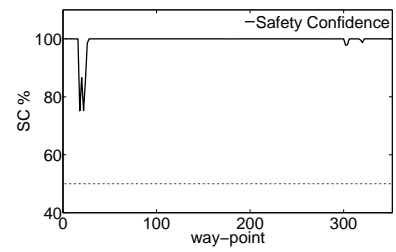

(f) $S C\left(S C_{\min }=70 \%\right.$.)
Fig. 8: Comparison of $S C$ and $\beta$ for some of the paths depicted in Fig. 7 in the UTIAS arena.

how planning subject to more significant stability margins and safety confidence $\left(\beta_{\min }=0.20\right.$ and $\left.S C_{\min }=90 \%\right)$ tends to result in longer (in yellow) routes, an intuitive outcome.

The result of planning based on the lowest allowable $\beta_{\min }=0.05$ and $S C_{\min }=50 \%$ (depicted in black in Fig. 7a and $7 \mathrm{~b}$ respectively) are found to be quiet coincidental. These two trajectories are going through (A) and passing directly over the central hill (C). Although the planning based on $\beta_{\min }=0.05$ ensures that instant value of $\beta$ are always larger than the minimum value, $\beta_{\mu}$ is found to be negative over the more challenging section, hence resulting in an $S C<50 \%$ i.e. a high risk for a tip-over instability as illustrated in the way-points around 150 in Fig. 8b. This would not represent a dangerous situation when planning based on e.g. $S C_{\min }=50 \%$, as the planner will reconfigure the robot so that it fulfils the minimum safety confidence.

Planning based on more comfortable $\beta_{\min }=0.10$ and $S C_{\min }=70 \%$ produced some interesting results. With $\beta_{\min }=$ 0.10 the planner could not find a trajectory through the front section (A) and resorted to move up towards (B), eventually finding a path via (D) to the goal. On the other hand, the planner with $S C_{\min }=70 \%$ considered the front section (A) feasible and found a shorter path which goes straight up to the middle of the arena and then coincide with the path with $\beta_{\min }=0.10$ in the final stages in the area labelled as (D). Looking at Fig. 8e around way-point 25 it is seen how $\beta$ around (A) is less than $\beta_{\text {min }}=0.10$, revealing the reason why planning based on $\beta_{\min }$ would not consider this area traversable. Looking at the value of $S C$ in Fig. $8 \mathrm{f}$ confirms that although $\beta$ is less than $\beta_{\min }=0.10$ around (A), safety confidence is larger than $70 \%$ and the planner regards this region as comfortably stable to plan over. This example shows how planning based on statistical data instead of the instant values can result in more effective and at the same time safer routes. The overall length of the trajectories illustrated in Fig. 7a and $7 \mathrm{~b}$ are summarised in Table I.

\begin{tabular}{|c|c|c|c|}
\hline$\beta_{\min }$ & length $(\mathrm{m})$ & $S C_{\min }$ & length $(\mathrm{m})$ \\
\hline 0.05 & 39.6184 & $50 \%$ & 39.0450 \\
\hline 0.10 & 43.4073 & $70 \%$ & 41.8475 \\
\hline 0.20 & 54.9470 & $90 \%$ & 53.0440 \\
\hline
\end{tabular}

TABLE I: Overall length of paths shown in Figure $7 \mathrm{a}$ and $7 \mathrm{~b}$.

\section{CONCLusion ANd Future Work}

This article presents a strategy for motion planning with stability uncertainty over rough terrains. The algorithm is able to exploit information gained from a statistical stability analysis to plan safe and effective routes under the presence of uncertainty in robot kinematics, terrain model and localisation on the terrain. Simulation results in an indoor rescue arena and an outdoor rover testing facility demonstrate how planning based on statistical safety metrics can result in safer and more effective routes when compared to a deterministic stability planning approach. The problem of global optimality in the decision-making navigation problem under stability and state uncertainty is left for future study.

\section{REFERENCES}

[1] C. Paulo and N. Urbano, "Path-following control of mobile robots in presence of uncertainties," IEEE Transactions on Robotics, vol. 21, no. 2, pp. 252-261, 2005.

[2] R. Gill, D. Kulic, and C. Nielsen, "Robust path following for robot manipulators," in IEEE/RSJ International Conference on Intelligent Robots and Systems, Tokyo, Japan, November 2013, pp. 3412-3418.

[3] K. Iagnemma and S. Dubowsky, Mobile Robot in Rough Terrain. Berlin, Germany: Springer Tracts in Advanced Robotics, 2004, vol. 12.

[4] M. Norouzi, J. V. Miro, and G. Dissanayake, "A statistical approach for uncertain stability analysis of mobile robots," in Proc. IEEE International Conference on Robotics and Automation, Karlsruhe, Germany, May 2013, pp. 191-196.

[5] _ - "Planning stable and efficient paths for articulated mobile robots on challenging terrains," in Proc. Australasian Conference on Robotics and Automation, UNSW, Sydney, Australia, December 2013, p. 10.

[6] E. Papadopoulos and D. A. Rey, "The force angle measure of tipover stability margin for mobile manipulatiors," Vehicle System Dynamics, vol. 33, no. 1, pp. 29-48, January 2000.

[7] C. H. Tong, D. Gingras, K. Larose, T. D. Barfoot, and E. Dupuis, "The canadian planetary emulation terrain 3D mapping dataset," The International Journal of Robotics Research, vol. 32, no. 4, pp. 389395, 2013.

[8] Y. Liu and G. Liu, "Interaction analysis and online tip-over avoidance for a reconfigurable tracked mobile modular manipulator negotiating slopes," IEEE/ASME Transactions on Mechatronics, vol. 15, no. 4, pp. 623-635, 2010.

[9] R. Smith, "Open dynamics engine, (http://www.ode.org/)," 2005. [Online]. Available: http://www.ode.org/

[10] R. Siegwart and I. R. Nourbakhsh, Introduction to Autonomous Mobile Robotos. The MIT press, 2004.

[11] Rubinstein and R. Y, Simulation and the Monte Carlo Method. New York: Wiley, 1981.

[12] S. J. Julier and J. K. Uhlmann, "Unscented filtering and nonlinear estimation," Proceedings of the IEEE, vol. 92, no. 3, pp. 401-422, 2004. 